\title{
ORL SCHEME FOR WIMAX AND LTE SYSTEM WITH CONVOLUTIONALLY CODED SPATIAL MULTIPLEXING
}

\author{
Wu Yin \\ School of EECE, University of Newcastle \\ Newcastle Upon Tyne, NE1 7RU, UK \\ E-mail:wu.yin@ncl.ac.uk \\ Pei Xiao, Colin Cowan \\ Institute of ECIT, Queen's University Belfast \\ Belfast, BT3 9DT, UK \\ E-mail: \{pei.xiao, c.cowan\}@ecit.qub.ac.uk \\ Shaoyang Liu, Qinfei Huang \\ School of Electronic Science \& Technology \\ National University of Defence Technology, China \\ E-mail: huang.qinfei@ nudt.edu.cn
}

\begin{abstract}
In 4G systems such as Wimax and LTE, system performance suffers from interferences and lack of low complexity algorithms for interference cancellation as well as utilization of optimal resource. In this paper, we propose a PCINR and EM based optimal resource allocation scheme to improve the performance of $4 \mathrm{G}$ systems and compensate the performance loss due to spatial correlation and interferences. Furthermore, the SIC technique is employed to mitigate interferences. The performance of the proposed system is evaluated by computer simulations and is shown to outperform the conventional schemes.
\end{abstract}

\section{KEY WORDS}

Resource allocation, WiMAX, LTE.

\section{Introduction}

There has been remarkable development in mobile wireless communications in this decade. Multiple output and input techniques (MIMO) and adaptive antenna system (AAS) techniques have been widely employed in $4 \mathrm{G}$ systems, e.g., worldwide interoperability microwave access (Wimax) and long term evolution (LTE) systems. However, there still exist some important issues to be resolved in the physical layer design of $4 \mathrm{G}$ systems.

It has been reported that carrier frequency offset which destroys the orthogonality between subcarriers and leads to inter-carrier interference [1] can deteriorate system performance significantly. In addition, in $4 \mathrm{G}$ systems, spatial correlation has more impact on the uplink than on the downlink [2]. Interference cancellation (IC) technique has been recommended as an efficient solution to tackle this problem.

In $4 \mathrm{G}$ systems, optimal resource allocation (ORL) can be implemented by adaptive modulation and coding (AMC) as well as with channel state information at the transmitter (CSIT). Precoding (beamforming) is an alternative technique that can effectively utilize optimal resource to maximize throughput and improve transmission link quality. CSIT plays a pivotal role in either ORL based or precoded closed-loop systems.

Physical carrier to interference-plus-noise ratio (PCINR) [3] and effective carrier to interference-plus-noise ratio (ECINR) are the two useful measurements to indicate the underlying channel condition, and they have been used in Wimax and LTE systems as feedback information from mobile terminals to base station. For simplicity, only PCINR is considered in this paper.

The tradeoff between computational complexity and system performance is a critical issue for $4 \mathrm{G}$ systems. Conventional optimization methodologies have prohibitive computational complexity which prevents them from practical implementation. Eigenvalue mode (EM) has been regarded as an efficient way to achieve desirable performance with reasonable complexity. For 
this reason, we develop an EM based algorithm in this paper.

It is well known that wireless communication systems are interference limited, i.e. the throughput and quality are affected dramatically by interferences such as multiuser interference (MUI), inter-symbol interference (ISI) and spatial correlation. VBLAST (Vertical-Bell Laboratories Layered-Space-Time) [4] can achieve good performance by successive interference cancellation algorithm (SIC) with low computational complexity. It is a technique that selects one received layer with the highest signal noise ratio (SNR) and then remove the relevant layer by SIC till the last layer is detected. However, the disadvantage of VBLAST is the lack of diversity for transmission link. To tackle this problem, parallel convolutional coding (PCCC) coded BLAST, which is also regarded as convolutional turbo coding (TBLAST), can be employed to compensate the diversity loss in VBLAST.

Nevertheless, the ORL and interference cancellation techniques have not been considered in the current Wimax and LTE standards. This paper provides a feasibility study for utilizing the ORL and interference cancellation on the transmitter and receiver side, respectively. It is reasonable to believe that the results obtained from this study are of direct relevance to the future version of $4 \mathrm{G}$ standards.

The rest of this paper is organized as follows. In Section 2 , the TBLAST algorithm, eigen mode and ORL techniques are reviewed. In Section 3, the system model and the proposed algorithms are presented, and the theory of the proposed EM and ORL based schemes are described. In Section 4, we present the simulation results to demonstrate the effectiveness of the proposed algorithms. Finally, conclusions are drawn in Section 5.

\section{Literature Review}

VBLAST, which has been regarded as a breakthrough in wireless communications, can achieve large capacity. However, the price to pay is the reduced transmission reliability due to lack of diversity that will deteriorate bit error rate (BER) performance. TBLAST, which has been widely used since its invention by Sellathurai and Haykin [4], has been considered in practical wireless communication systems to improve BER performance.

\subsection{Review of PCINR}

Channel estimation and resource optimisation are the two main issues that can determine the physical layer system performance in both LTE and Wimax systems. Recently, several equipment vendors have issued a proposal that involves PCINR and power allocation [5,6] in Wimax Forum. Channel estimation is based on PCINR. CSIT or
PCINR information can be in the form of precoding matrix index (PMI) in both Wimax and LTE systems.

It can be seen from [6] that PCINR is related to the Shannon capacity, which will be discussed in details in the following section. Our proposed algorithms with eigen mode depend on the analysis of PCINR. In [7,8], compensation booting was considered for ORL, and AMC was used to improve system performance in the wireless communication systems. However, there have been lack of specific methodologies and applications in $4 \mathrm{G}$ systems.

In Wimax beamforming or LTE precoding techniques, eigen mode based techniques can be considered for weight calculation or code book selection in the precoding of transmission in base station. ORL can be faciliated by fast feedback channel (FFB) and CSIT in the closed-loop system.

\subsection{Review of SVD Scheme}

The principle of SVD (singular value decomposition) can be described briefly as the following equation:

$$
\mathbf{H} * \operatorname{diag}(\boldsymbol{\lambda})=\mathbf{v} * \operatorname{diag}(\boldsymbol{\lambda})
$$

where $\mathbf{H}$ is a normalised $n \times m$ complex channel matrix, each element of which has zero mean and unit variance. The vector $\lambda$ contains the eigenvalues sorted in descending order, $\quad \lambda_{1} \geq \lambda_{2} \geq \lambda_{n}$ i.e.,

They can be regarded as the channel gain in wireless propagation, and the maximum eigenvalue is equal to the power of the channel. The relevant eigenvector are:

$$
\mathbf{v}=\left[\mathbf{v}_{1} \mathbf{v}_{2} \ldots \mathbf{v}_{\mathbf{n}}\right]
$$

which can be utilized to form precoding matrix in the MIMO systems. The above equation provides a basis for the low complexity least square (LS) algorithm, which is widely employed in the wireless communication systems such as MIMO and smart antenna beamforming (BF) to achieve the maximum SNR.

\section{Proposed Schemes}

In the proposed system, the Minimum mean square error (MMSE) and Log-Map algorithms are employed for channel estimation and decoding, respectively. In addition, SIC and ORL are also employed to improve the system performance.

In Wimax beamforming or LTE precoding techniques, eigenvalue base techniques can be considered for weight calculation or code book selection in the precoding of transmission in base station. The conventional ORL 
algorithms depend on utility functions such as SINR, BER performance or system capacity. The cost function of signal to interference and noise ratio (SINR) can be expressed as [9,10]:

$$
\gamma\left(p_{i}\right)=\frac{a_{i} p_{i}}{\sum_{j \neq i} b_{j} p_{j}+n_{i}}=\left\{\frac{\left\|v_{i} p_{i} \mathbf{H}_{i}\right\|^{2}}{\sigma^{2}\left\|v_{i}\right\|^{2}+\sum_{j \neq i}\left\|v_{k_{j}} p_{j} \mathbf{H}_{j}\right\|^{2}}\right\}
$$

where $\left\{a_{i}\right\}$ denotes the processing gain; $\left\{b_{j}\right\}$ denotes the fading channel gain; $\left\{n_{i}\right\}$ is a zero mean AWGN; $v_{i}$ is the nulling vector and $\left\{p_{i}\right\}$ is the power allocated to individual substream in the transmitter.

In 4G systems, the maximum signal to noise ratio (MSNR) based precoding scheme is equivalent to the maximum SINR based scheme. The system capacity can be presented as follows:

$$
\begin{aligned}
C=\log (\operatorname{det} & \left.\left(\mathbf{H}_{i} \mathbf{Q}_{i} \mathbf{H}_{i}^{H}+\sigma^{2} \mathbf{I}\right)\right) \\
& =\sum_{n=1}^{N} \log \left(1+\operatorname{SINR}_{k, n}\right)
\end{aligned}
$$

where ()$^{H}$ presents the complex conjugate operation; SINR denotes signal to interference and noise ratio; $\mathbf{H}$ is a normalised $m \times n$ complex-valued channel matrix as defined earlier, $\mathbf{Q}$ is the covariance matrix of the information data bits and the $\sigma^{2}$ denotes the variance of Additive White Gaussian Noise (AWGN).

The ergodic capacity of MIMO systems, which is defined as a maximum average mutual information that depends on the complex Gaussian identical independent distribution (i.i.d) channel, can be expressed by the following equations with perfect CSI at receiver and no CSI information in transmitter:

$$
\begin{aligned}
f\left(p_{i}\right) & =E_{H}\left[\log _{2}\left[\operatorname{det}\left(\mathbf{I}+\frac{\rho}{m} \mathbf{H Q H}^{\mathbf{H}}\right)\right] \mathrm{b} / \mathrm{s} / \mathbf{H z}\right] \\
& =\frac{1}{m} \sum_{i=1}^{m} \log \left(1+\frac{p_{i} \lambda_{i}}{\sigma^{2}}\right)
\end{aligned}
$$

where ${ }^{\rho}$ denotes the average SNR. The min-max [10] equation with power constraint is described as:

$$
\max \left[f\left(p_{i}\right)\right]-\sum_{i=1}^{m} k_{i} g_{i}\left(p_{i}\right)
$$

The power allocated to each individual substream and constraint can be expressed in the form of eigenvalue as

$$
g_{i}\left(p_{i}\right)=P_{c}-k_{i} \sum_{i=1}^{m} p_{i} \lambda_{i}
$$

where $P_{c}=$ trace $\left(\mathbf{Q Q}{ }^{H}\right)$ is the power constraint; $\left\{k_{i}\right\}$ are the lagrangian coefficient.

Subsequently, we can obtain the first order of derivative as:

$$
\begin{aligned}
e_{i}= & \frac{d L\left(p_{i,}, k_{i}, r_{i}\right)}{d p_{i}} \\
= & \frac{d f\left(p_{i}, r_{i}\right) \operatorname{TABLEgI}\left(p_{i}, r_{i}\right)}{\text { SIMULATION } k_{\text {ENVIRONMENT }}}
\end{aligned}
$$

$\begin{array}{ll}\text { Simulation model } & \text { Mento Carlo } \\ \text { Transmit antenna } & \text { 3 elements } \\ \text { Receive antenna } & \text { 6 elements } \\ \text { Fading channel } & \text { Rayleigh. Jakes model } \\ \text { Doppler frequency } & 20 \mathrm{~Hz} \\ \text { Encoder \& rate } & \text { CTC 1/2 } \\ \text { Data modulation } & \text { OFDM QPSK } \\ \text { Decoding algorithm } & \text { Log-Map, } \\ & \text { extrinsic information transfer (EXIT) } \\ \text { Constraint length L } & 4,6 \\ \text { Feedback polynomial } & 111(\mathrm{~L}=4), 11011(\mathrm{~L}=6) \\ \text { Feedforward } & 101(\mathrm{~L}=4), 11001(\mathrm{~L}=6) \\ \text { polynomia } & 6 \\ \text { Number of iterative } & \text { Perfect known } \\ \text { CSI } & \text { PCCC, Closed-loop } \\ \text { System } & \end{array}$

The solution can be derived by setting (5) to zero, and it can be applied to the transmitter to optimize resource allocation.

\section{Simulation Results}

In this section, the performance of the proposed system is evaluated by computer simulations and numerical results are given to demonstrate the effectiveness of the proposed schemes. The simulation parameter setting is tabulated in Table I, where the polynomial equation can be described as:

$$
G(D)=\left(1, \frac{1+D+D^{2}+D^{3}}{1+D^{2}+D^{3}}\right)
$$




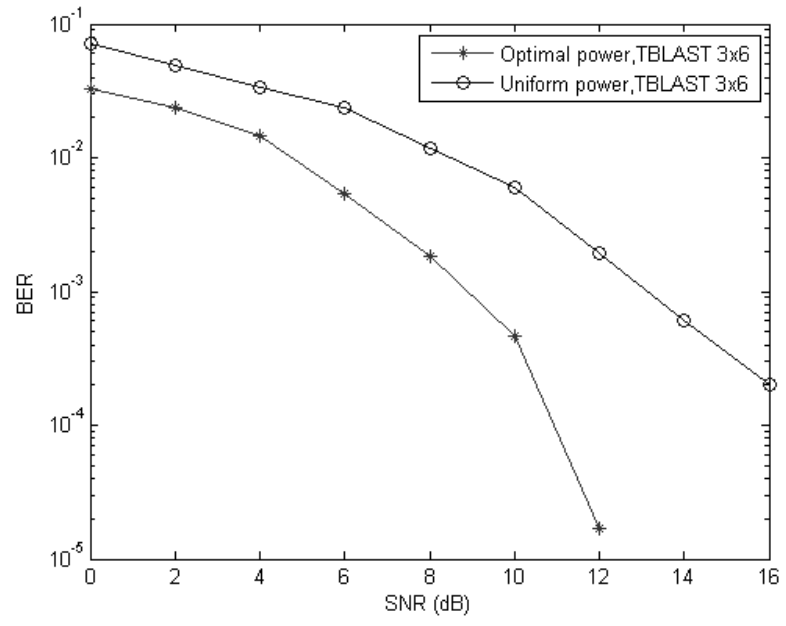

Fig.1 Performance comparison: ORL versus equal power allocation

Fig. 1 demonstrates the clear advantage of using the proposed ORL scheme compared to the equal power scheme. A gain of $4.5 \mathrm{~dB}$ has been observed at target $\mathrm{BER}=10^{-3}$, and the performance improvements become more obvious as SNR increases.

\section{Conclusion}

In this paper, a closed-loop SIC, eigen mode and PCINR based system with optimal power allocation has been proposed and evaluated by means of simulations. Results show that the proposed ORL scheme achieves a significant improvement in system performance by effective interference mitigation and the utilization of optimal resources.

\section{References}

[1] R. Raghunath, A. Chockalingam, "SIR Analysis and Interference Cancellation in Uplink OFDMA with Large Carrier Frequency and Timing Offsets", Proc. IEEE Wireless Communications \& Networking Conference, Kowloon, China, 2007.

[2] B. Hamid, L. Jonathan, P. Jules, U. Raner, "A study on UL potential issues", Wimax Forum, Technical working group, 2008, www.wimaxforum.org

[3] J. Huang, N. Yanxin, "Physical carrier to interference-plus-noise ratio techniques for wideband wireless communications networks", Cisco Technology, INC, USPC Class: 375346.

[4] G. Foschini, "Layered Space-Time Architecture for Wireless Communication in a Fading Environment When Using Multi-Element Antennas", Bell Labs Technical Journal, vol. 1, no. 2, autumn 1996.
Correlation

[5] M. Sellathurai, S. Haykin, "Turbo-BLAST for wireless communications: theory and experiments", IEEE Trans. on Sig. Proc., vol. 50, no 10, pp. 2538 2546, Oct. 2002.

[6] "DL MIMO PCINR", Beceem, Alvarion, Runcom. Arraycomm, Nextwave, Fujitsu, Clearwire and Altair, Wimax Forum, 2008.

[7] H. Vesa and K. Heikki "SINR Estimation for power control in systems with transmission beamforming", IEEE Communication Letters, vol. 9, no. 10, Oct. 2005.

[8] "Mimo precoding enabling spatial multiplexing, power allocation and adaptive modulation and coding", USPTO Application \#: 20080080634, 2008.

[9] H. Simon, M. Michael, "Modern wireless communications" Publisher: Prentice Hall, March 2004.

[10]E.A. Jorswieck, H. Boche. "Transmission strategies for the MIMO MAC with MMSE receiver: average MSE optimization and achievable individual MSE region", IEEE Trans. Sig. Proc. vol. 51, pp. $2872-$ 2881, Nov. 2003. 